\title{
Problemática de la equivalencia translémica español-italiano a través de una novela de Manuel Vázquez Montalbán
}

\author{
ROSA MARÍA RODRÍGUEZ ABELLA
}

\section{Introducción}

A Miguel Martínez-Lage (2007: 216-217), 'su larga práctica traductora le lleva a sostener que el traductor es ante todo lector,

\begin{abstract}
lector privilegiado y lector responsable, ya que de su lectura, de su movimiento interpretativo, han de emanar todas las demás lecturas. El traductor no puede abrir, pero sobre todo no puede cerrar el espectro de las ambigüedades del original. Su lectura ha de cifrarse en la reproducción no de lo que pone en el original, sino de lo que el original dice, de modo que una vez traducido siga diciendo lo mismo, al margen de lo que ponga, y todas las interpretaciones del original sigan siendo posibles en el texto traducido.
\end{abstract}

Desde su punto de vista (ibídem, 216), al enfrentarse a un nuevo trabajo traslaticio, el traductor ha de realizar, en primer lugar, una lectura profunda y responsable del texto $\mathrm{y}$, a continuación, ha de empaparse "del contexto lingüístico-cultural en que se mueve el autor" que está traduciendo. Para Martínez-Lage, conocer el contexto de la obra deviene un elemento indispensable para, como sostenía también Valentín García Yebra (1994: 258), poder "decir todo lo que dice el texto original".

Por otra parte, la obra montalbaneana, como sintetiza muy acertadamente Colmeiro (2013: 20).

\footnotetext{
${ }^{1}$ Miguel Martínez-Lage (Pamplona, 1961-Almeria, 2011), obtuvo, entre otros reconocimientos, el Premio Nacional de Traducción en 2008 por su versión de la Vida de Samuel Johnson de Samuel Boswel.

${ }^{2}$ Según el traductor navarro, "en el fondo, la cuestión es sencilla: se trata de ponerse en el pellejo del lector de la novela en su versión original -y, llegado el caso, hablar con un lector de la novela en versión original para saber qué percibe, qué acusa- y desmenuzar entonces todos los efectos que esa novela haya podido producir en su sensibilidad para reproducir esos mismos efectos, a veces generando otros mecanismos, de acuerdo con determinadas convenciones y sometiéndose siempre a la signatura del autor (incluso si contraviene determinados usos), para que esos efectos sean análogos en la lengua de llegada" (2007: 218).
} 
[...] atraviesa las fronteras estrictas de los géneros literarios y subvierte categorías tradicionales de cultura alta y baja, combinando lo ficcional y lo histórico, el ensayo y la novela, el periodismo y la literatura, y temas tan diversos y aparentemente tan alejados entre sí como las gastronomía y el erotismo, el fútbol y el nacionalismo, la sexualidad y la política, los medios de comunicación de masas y la memoria histórica.

Ciertamente la rica y variada presencia de referentes culturales en la saga carvalhiana puede complicar la labor del traductor. Es más, a nuestro modo de ver, para solventar las dificultades traslaticias que plantean los textos tan marcados culturalmente es casi imprescindible que la cultura del traductor abarque la materia tratada en la obra que está traduciendo. Porque, a veces, descifrar el sentido del texto original se convierte en un ejercicio de hermenéutica literaria que rebasa la habitual labor de transferencia lingüística y cultural que suele implicar la traducción de un texto. ${ }^{3}$ Así pues, en estas líneas, utilizando como corpus de referencia la traducción al italiano de Asesinato en el Comité Central (1981), nos planteamos reflexionar sobre las dificultades traslaticias que comportan, en particular, los referentes culturales pertenecientes al ámbito del patrimonio cultural.

\section{Asesinato en el Comité Central}

En esta crónica carvalhiana, el protagonista acude por encargo a la Villa y Corte para investigar el asesinato del secretario general del PCE. En este viaje a la capital y "al interior del PCE" (Pradera 1981; Arroyo 1983) asistimos a toda clase de peripecias investigativas, eróticas, gastronómicas, etc.

Sin duda, el núcleo argumental, como sugiere Javier Pradera (1981), presenta "una idea con deslumbradoras posibilidades cinematográficas":

\section{$-$}

${ }^{3}$ Además, como es sabido, el idiolecto montalbaniano se caracteriza por el uso esporádico del catalán. Característica esta que puede dificultar ulteriormente el trabajo del traductor.

${ }^{4}$ Assassinio al Comitato Centrale se publica en la Sellerio editore Palermo tan solo tres años después del texto original (1984). La versión italiana es obra de Lucrezia Panunzio Cipriani. Por otra parte, esta es la primera y única incursión de esta traductora en el universo Carvalho. En 2005 la editorial Feltrinelli de Milán publica también esta novela, pero se sirve de la misma traducción.

${ }_{5}^{5}$ De hecho, en 1982 Vicente Aranda dirige Asesinato en el Comité Central con Patxi Andión y Victoria Abril como protagonistas. 
el secretario general del Partido Comunista de España es asesinado en una reunión del Comité Central, con el agravante de la nocturnidad artificial proporcionada por un apagón de luz intencionado, y los camaradas de la víctima encargan al detective privado Pepe Carvalho - ex comunista y ex agente de la CIA, acreditado profesionalmente por los éxitos conseguidos en Tatuaje, La soledad del manager y Los mares del Sur - la investigación del caso. Para colmo, esta réplica hispánica de los grandes misántropos y escépticos que pueblan la novela negra americana tiene que competir en su trabajo con un policía en cuya figura hasta los más arcangélicos querubines encontrarían sorprendentes parecidos con el comisario Conesa (ibídem).

Por otra parte, a medida que avanza la narración se va perfilando un retrato muy personal de la sociedad capitalina. De hecho, la narración se localiza básicamente en Madrid, en los últimos años de la Transición, antes del triunfo socialista en las Elecciones Generales del 25 de octubre de 1982.

En cualquier caso, como veremos al final, "detrás de todo está siempre el poder del dinero, una realidad cada vez más literal, más descarnada" (García Montero, 2012: 11).

\section{Peritexto y traducción}

En cuanto a las formas constitutivas externas más interesantes desde el punto de vista traductológico, el título es sin duda uno de los elementos peritextuales más visibles y además, como apunta Divinsky (citado en Guerriero, 2013: 5), "un título no hace que un libro se venda, pero hace

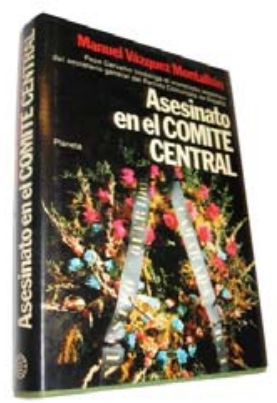
que el candidato a comprarlo lo levante de la mesa". En nuestro caso, el título alude claramente al contenido del texto: un asesinato y al lugar del hecho: el Comité Central. Por tanto, según Genette (2001: 70), nos hallamos ante un título que funciona como indicador temático y descriptor de la naturaleza del texto.

En la edición italiana, en una casi total simetría con el paratexto original, se opta por la traducción literal del título de la obra: Assassinio al Comitato Centrale. 
Un elemento peritextual que sí se altera es la imagen de portada del libro. En la edición original de Planeta aparece una corona de flores en cuya banda encontramos la leyenda: A nuestro querido camarada R.I.P., donde la referencia al fallecimiento de un miembro del partido comunista es evidente. Se establece, por tanto, una perfecta conjunción entre los signos

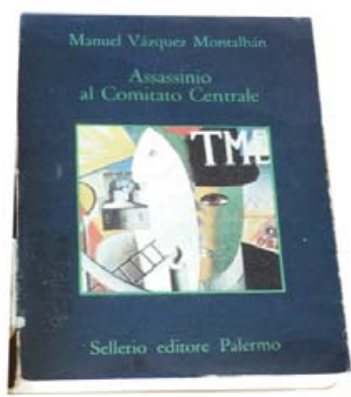
verbales del título y la imagen de cubierta.

Sin embargo, en la edición italiana se pierde por completo esta alusión. De hecho, como imagen de portada se nos propone un detalle del cuadro de Malevich Un inglés en Moscú (1914). En consecuencia, desaparece el vínculo entre elementos verbales e iconográficos presente en el texto origen.

«... nos hemos liberado de la fe ciega acientífica, y se ha reforzado en nosotros esa fe a la que se refería Marx cuando decía que los comunistas son capaces de "asaltar los cielos". Cuando se enfría esa fe, cuando se empieza a dudar, cuando se hace uno un descreído, empieza uno a dejar de ser comunista. Ésta es la verdad."

IRENE FALCÓN

(citado por JORGE SEMPRÚN en Autobiografía de Federico Sánchez)

Pero la muerte muestra de repente que la sociedad real mentía.

Georges Bataille

(Teoría de la religión)

Fig.1. Epígrafes en Asesinato en el Comité Central.

Otros elementos constitutivos externos característicos del paratexto original son, en el siguiente orden, la dedicatoria autoral, dos epígrafes 
alógrafos, una nota del autor y la disolución de la división en capítulos. En el TM se altera el orden de algunos elementos peritextuales, de tal manera que primero se sitúan los dos epígrafes, después la dedicatoria y, en último lugar, la nota. En cambio, se mantiene idéntica la fragmentación del texto en secuencias.

Sorprende esta alteración del orden paratextual, puesto que la dedicatoria suele ser un escrito o nota que se pone en un libro al principio, esto es, suele preceder al texto que simbólicamente es dedicado. ${ }^{6}$

En cuanto a los dos epígrafes alógrafos, no cabe duda de que estos contribuyen a orientar al lector, de hecho, para Montalto (1989: 21-22), el epígrafe:

[...] rappresenta il primo contatto stabilito con il lettore, diventa il primo tramite, la prima comunicazione fra l'autore e il suo destinatario. La sua scelta manifesterà all'epigrafatario l'indirizzo, l'intenzione, lo spirito, l'umore, talvolta il tema dell'opera; [...] avrà la funzione di sollecitare nel lettore il nascere di una tensione nei confronti dell'opera, di prepararlo emotivamente all'atmosfera della stessa, attivando quei sentimenti che serviranno per una corretta lettura.

Esto es, el epígrafe funciona como una llamada de atención al lector, sugiriéndole o apuntándole posibles claves interpretativas sobre el contenido y sentido del texto que va a leer. Montalto (1991: 14) destaca también que:

il fascino maggiore dell'epigrafe consiste proprio nella sua varietà e nelle sue vastissime opportunità di relazioni che l'autore vuole stabilire con il fruitore di essa; a volte si tratta di un sottile gioco ironico o polemico o ammiccante, di un'allusione ambigua o insinuante, altre deve acrescere la sensazione, l'emozione. Non è raro il caso in cui lo scrittore si serve di una citazione desiderando dal suo lettore un collegamento non solo con la stessa, ma con tutto il testo da cui è stata tolta.

En el caso que nos ocupa, no hay que olvidar que el propio MVM, como confiesa a Blanco Chivite (1992), sentía una especial preferencia

\footnotetext{
${ }^{6}$ Sobre la colocación y uso de la dedicatoria, Sabia (2005) incide en que: "La dedicatoria es una práctica por medio de la cual el autor homenajea a una persona o a un grupo de personas haciendo figurar, al inicio de su obra, el nombre del destinatario de su homenaje. Suele aparecer inmediatamente después de la página que reproduce la portada con el título de la obra y el nombre del autor".
} 
por esta novela, aunque reconocía también que le había costado mucho escribirla ${ }^{7}$ :

era muy difícil escribir una novela sobre el PCE, dentro del PCE, sin ser disidente, sin hacerlo como el clásico disidente que se ha marchado y al mismo tiempo tratando de explicar qué coño está pasando y qué coño va a pasar, porque la novela está publicada antes de la gran liquidación, antes de la merienda de rojos. (Citado en Saval, 2004:167) ${ }^{8}$

Con todo, MVM siempre sostuvo que la obra era "básicamente una novela de aventuras y también una crítica al sentido religioso de la militancia" (Canals, 1981).

Por último, por lo que se refiere a la nota del autor, esta encierra claramente un matiz aclaratorio por parte de MVM acerca de la naturaleza ficcional de la historia que nos va a narrar.

Esta acotación adquiere especial relevancia, porque pese al aparente distanciamiento, no cabe duda de que nos hallamos ante un narrador comprometido y que la obra refleja perfectamente el contexto sociopolítico en que fue escrita9. Conocida es, por ejemplo, la polémica suscitada por Santiago Carrillo cuando se publica esta novela ${ }^{10}$. En efecto, el dirigente comunista ve en Fernando Garrido su trasunto literario y así lo sostiene en el Epílogo conmemorativo del $25^{\circ}$ aniversario de Carvalho:

el escritor escogió la forma de la novela policiaca para elaborar un libro político. [...] A nadie -ni al autor- podrá extrañar, pues, el reconocimiento de que cuando se publicó lo sentí como una auténtica puñalada. La que recibió físicamente Fernando Garrido iba dirigida políticamente contra mí (1997: 295).

\footnotetext{
${ }^{7}$ Sobre la conflictiva relación de MVM con el PCE, véase Tyras (2003: 26-36).

${ }^{8}$ Saval (2004: 168) sostiene también que "más allá de lo anecdótico, Asesinato en el Comité Central, es posiblemente la novela donde el escritor haya incluido más referencias personales, sus propias creencias, anécdotas (como la de que en una reunión clandestina dijo que los comunistas de finales de los años cincuenta no durarían ni un cuarto de hora en el monte), etc.".

${ }^{9}$ En 1981 el Partido Comunista de España estaba sumido en una profunda crisis institucional. En el X Congreso del PCE Santiago Carrillo es reelegido Secretario general pero se asiste también a la escisión interna entre carrillistas y renovadores.

${ }^{10}$ En cualquier caso, ante el fracaso electoral del PCE en 1982 se producirá una fuerte lucha interna en el partido y Carrillo se verá forzado a dimitir.
} 
Sea como fuere, ciertamente el novelista nos presenta el retrato de un medio bien conocido por él. Es más, hasta el propio Carvalho se siente autorizado a emprender ese viaje a la capital y "al interior del PCE". De hecho, el diálogo que Carvalho mantiene con Santos Pacheco así parece confirmarlo:

- ¿Por qué yo?

- Porque usted es un ex comunista. Porque usted sabe qué somos, cómo somos, de dónde venimos, adonde vamos (33-34).

En cuanto a la traslación de esta nota, constatamos que se verifican algunas imprecisiones y un error patente ${ }^{11}$.

\begin{tabular}{|c|c|}
\hline NOTA DEL AUTOR & Nota dell'Autore \\
\hline $\begin{array}{l}\text { Ante la previsible y perversa in- } \\
\text { tención de identificar los personajes } \\
\text { de esta novela con personajes reales, } \\
\text { el autor declara que se ha limitado a } \\
\text { utilizar arquetipos, aunque reconoce } \\
\text { que a veces los personajes reales nos } \\
\text { comportamos como arquetipos. }\end{array}$ & $\begin{array}{l}\text { Davanti alla prevedibile e subdola } \\
\text { intenzione di identificare i personaggi } \\
\text { di questo romanzo con personaggi } \\
\text { reali, l'Autore dichiara che si è } \\
\text { limitato a utilizzare degli archetipi, } \\
\text { anche se riconosce che a volte i } \\
\text { personaggi reali si comportano come } \\
\text { archetipi. }\end{array}$ \\
\hline $\begin{array}{l}\text { ARQUETIPO: Tipo soberano y } \\
\text { eterno que sirve de ejemplar y modelo } \\
\text { al entendimiento y a la voluntad de } \\
\text { los hombres. } \\
\text { (Del Diccionario de la Real Aca- } \\
\text { demia) }\end{array}$ & $\begin{array}{l}\text { ARCHETIPO: Immagine sovrana ed } \\
\text { eterna che serve da esempio e da } \\
\text { modello all'intelletto e alla volontà } \\
\text { degli uomini. } \\
\text { (Dal Dizionario della Real } \\
\text { Academia) }\end{array}$ \\
\hline
\end{tabular}

Comprobamos que al trasladar la preposición castellano ante (utilizada en el TO en sentido figurado) por el adverbio de lugar italiano davanti (literalmente en castellano delante de), en el TM se produce un sin

\footnotetext{
${ }^{11}$ Una imprecisión innecesaria se verifica al trasladar el adjetivo castellano perverso por el italiano subdolo (literalmente. taimado), puesto que en italiano existe el adjetivo homólogo perverso, que presenta además una extensión semántica idéntica a la del castellano.
} 
sentido $^{12}$, efectivamente, la locución adverbial italiana equivalente es: $d i$ fronte $a$.

Se altera también el punto de vista presente en la secuencia discursiva original: a veces los personajes reales nos comportamos como arquetipos, al trasladarse al italiano por: a volte i personaggi reali si comportano come archetipi. Como se puede ver, en el TM se recurre a la técnica de la modulación, produciéndose un cambio de categoría de pensamiento. De hecho, en el TO el autor se incluye en la generalización que acaba de reseñar, sin embargo, en el TM se pierde este matiz que se hubiera podido mantener mediante la traslación: $\underline{\text { noi, personaggi reali, }} \underline{\text { ci comportiamo. }}$.

Estudio analítico de los referentes pertenecientes al ámbito del patrimonio cultural y su traducción al italiano

Dados los límites de extensión y contenido del presente estudio, circunscribiremos nuestro análisis a un único dominio cultural ${ }^{13}$, en concreto, nos ceñiremos al campo del patrimonio cultural.

En este ámbito incluimos los problemas de traducción ocasionados por las referencias físicas o ideológicas que comporta una cultura, por consiguiente, los personajes reales o ficticios, los acontecimientos históricos, las obras y movimientos artísticos, el folklore, el cine, la música, los bailes, los juegos, las festividades, los monumentos emblemáticos, la comida, la bebida, etc.

Además, ante la imposibilidad material de incluir todas las secuencias textuales que presentan inadecuaciones de traducción en el campo del patrimonio cultural, haremos una selección de los segmentos textuales más representativos.

\footnotetext{
$-$

${ }^{12}$ La técnica utilizada en este caso es la de la transposición, En este trabajo, como en otros precedentes (Rodríguez Abella, 2008, 2009, 2010, 2013), nos atenemos a la catalogación de técnicas de traducción propuesta por Hurtado Albir (2004: 269-271).

${ }^{13}$ Nos atenemos a la clasificación de ámbitos culturales propuesta por Molina (2006: 7982). Esta autora identifica cuatro dominios sensibles a las interferencias culturales: medio natural, patrimonio cultural, cultura social y cultura lingüística. Además, en un epígrafe aparte, afronta también la cuestión de las interferencias culturales, distinguiendo entre falsos amigos culturales e injerencias culturales.

${ }^{14}$ Véase Molina (2006: 81).
} 


\section{Patrimonio cultural}

\begin{tabular}{|c|l|}
\hline TO (p. 24) & \multicolumn{1}{|c|}{ TM (p. 25-26) } \\
\hline Biscuter no había tenido tiempo ni & Biscuter non aveva avuto tempo né di \\
de deslegañarse ni de ordenar mínima- & ripulirsi né di mettere un po' d'ordine \\
mente la mesa del despacho. & sul tavolo dell'ufficio. \\
$\begin{array}{c}\text { - ¿Desayuna aquí, jefe? Tengo unas } \\
\text { butifarras de perol de puta madre y unos }\end{array}$ & - Pranza qui capo? Ho delle salsicce \\
\hline fesols cocidos que sobraron de ayer. & $\underline{\text { fagioli lessi avanzati ieri. }}$ \\
\hline
\end{tabular}

Aunque no es nuestro propósito analizar aquí las diferentes funciones que el discurso gastronómico adquiere en la saga Carvalho, ${ }^{15}$ no cabe duda de que la cocina es un elemento caracterizador de la serie novelesca. ${ }^{16}$

En concreto, nuestro detective siente especial predilección por los gustos tradicionales, por las recetas regionales españolas, sobre todo, las de Cataluña, Galicia, Valencia, Murcia, etc. ${ }^{17}$ Es más, constatamos que hay una serie de productos, técnicas y platos habituales en casi todas las novelas de la saga. Entre los productos más recurrentes se encuentran, sin duda, las butifarras. En esta secuencia textual se trata de las butifarras de perol, esto es, de las butifarras de matanza. Nos hallamos pues ante un embutido que carece de referente y designación en la lenguacultura meta. Ante la dificultad que plantea la traslación de esta referencia cultural, la traductora opta por un doblete de técnicas, generalización y traducción literal. Así pues, en primer lugar, utiliza el hiperónimo salsicce y, en segundo lugar, opta por la técnica de la traducción literal, no obstante, incurre en un error al confundir con un topónimo el vocablo perol. Se hubiera podido evitar la inadecuación manteniendo la letra $-$

15 Aspecto destacado ya por varios especialistas en la obra montalbaneana, véase, por ejemplo, Colmeiro (1994: 186-193) y Tyras (2003: 118-119).

16 Para Newmark (2004: 137), por ejemplo, la comida representa "la expresión más delicada e importante de la cultura nacional".

${ }^{17}$ Como afirma el propio Vázquez Montalbán (2004: 8), la base de los gustos culinarios de Carvalho "la forma una materia esencial: el paladar de la memoria, la patria sensorial de la infancia. Por eso sus gustos fundamentales proceden de la cocina popular, pobre e imaginativa de España”. Esto es, "integra cocina catalana, cocina de autor de distintos restauradores de España y de diferentes extranjerías gastronómicas”. En definitiva, cocina y cultura mestiza que dificultan, sin duda, la labor del traductor. 
minúscula o trasladando el referente como salsicce catalane fresche, botifarra fresca, o bien, botifarra de perol, las dos últimas soluciones nos parecen las más adecuadas, visto que se trata de un término muy recurrente en toda la serie.

Aunque no se trata de un término marcado culturalmente, se produce también una inadecuación innecesaria al trasladar el verbo desayunar por el verbo italiano pranzare, literalmente en castellano almorzar, el término equivalente es fare colazione. ${ }^{18}$

En último lugar, aunque se trata de una expresión perteneciente al ámbito de la cultura lingüística, observamos que se altera el tenor del discurso al trasladar de puta madre por la expresión italiana la fine del mondo.

\begin{tabular}{|c|l|}
\hline TO (p. 25) & TM (p. 26) \\
\hline $\begin{array}{l}-¡ \text { Oiga qué dice este facha, jefe! } \\
i \text { Escuche! }\end{array}$ & $\begin{array}{l}\text { - Capo, senta che dice questo } \\
\text { minchione! Ascolti! }\end{array}$ \\
\hline
\end{tabular}

En este segmento, el vocablo facha, fruto del acortamiento del término italiano fascista, encierra una clara referencia ideológica para indicar que alguien es partidario de un movimiento político y social de carácter totalitario ${ }^{19}$. En cambio, minchione, literalmente en castellano gilipollas, capullo, según la definición del Treccani indica: "persona sciocca, priva di furberia, eccessivamente semplice e credulona; è soprattutto usato come titolo di spregio e di rimprovero", esto es, indica una persona tonta, excesivamente simple.

Por tanto, en italiano sería más adecuado recurrir al término fascista o fascistone, porque minchione introduce un matiz no presente en el TO.

\begin{tabular}{|l|cc|}
\hline TO (p. 37) & TM (p. 38 ) & \\
\hline Prefirió, pues, ir caminando hacia & Preferì andare a piedi fino alla \\
\hline
\end{tabular}

\footnotetext{
$-$

${ }^{18}$ De acuerdo con Bensoussan (1991: 20), consideramos que «el traductor debe sentarse en la mesa del autor, debe introducirse en él o en su obra, tiene que buscar pistas, indicios, huellas, intuiciones, tiene que profundizar todos los contextos necesarios».

${ }^{19}$ Efectivamente, Biscuter está escuchando por la radio qué opinan varios encuestados sobre la situación que se ha creado tras el asesinato de Garrido. Entre otras cosas, este encuestado comenta lo siguiente: "desde que murió Franco nos ha caído encima la plaga" (1997: 25).
} 
la Boquería a comprar dos kilos de escupiñas y pescado para hacer caldo. Luego rescató el coche del parking de La Garduña para irse a tomar un bacalao a l'hostal en el figón Pa i Trago, una casa de comidas cercana al mercado de San Antonio, donde los seres humanos civilizados pueden desayunar capipota con sanfaina desde las nueve de la mañana. pescheria per comprare due chili di frutti di mare e di pesce per fare la zuppa. Poi riprese la macchina dal posteggio della Garduña per andarsi a mangiare un filetto di baccalà al "Pa $\mathrm{i}$ Trago", una rosticceria vicino al mercato di Sant'Antonio, dove gli esseri umani civilizzati possono gustare peperonata con stufato dalle nove di mattina.

Como es sabido, la Boquería ${ }^{20}$, emblemático mercado de Barcelona, aparte de ser un lugar donde se pueden comprar todo tipo de productos frescos, es también una atracción turística mundialmente conocida. Por tanto, el recurso a la técnica de la particularización, es decir, el uso de un término más concreto, pescheria, literalmente en castellano pescadería, no nos parece acertado. De hecho, es de sobra sabido que en este famoso mercado no solo se compra y vende pescado, sino también muchos otros productos: carnes, verduras, etc. Por esta razón, consideramos que en esta secuencia hubiera sido más adecuado mantener el vocablo tal cual.

En cuanto a la denominación de la escupiña o venus verrucosa, en italiano la más común es tartufo di mare, aunque también se la conoce con otros nombres: cappa verrucosa, noce y venere tartufo, además de numerosas designaciones dialectales. En este caso, la traductora ha optado por una generalización, recurriendo al hiperónimo frutti di mare, literalmente en castellano moluscos. De todas maneras, se produce un falso sentido en el TM al no introducir el artículo partitivo del antes del término pesce. Es decir, Carvalho compra solo algo de pescado, no dos kilos de escupiñas y de pescado. También se produce una inadecuación en la traslación de la palabra caldo, el término equivalente en italiano es brodo y no zuppa, literalmente en castellano sopa.

Para la traducción del plato que consume Carvalho, la traductora opta por un doblete de técnicas: amplificación (castellano un bacalao $=$ italiano un filetto di baccalà) y elisión (castellano a l'hostal= italiano Ø), en

$-$

${ }^{20}$ El mercado de la Boquería fue inaugurado en 1840, el 19 de marzo, día de San José. Para más información sobre su origen, personajes que lo frecuentan, mitos, etc., véase Obiols, I. y P. Ferrer (2004). 
la traslación se elide asimismo el término figón.

Por lo que se refiere a la designación casa de comidas, en realidad, una rosticceria (literalmente en castellano rotisería) ${ }^{21}$ es una tienda donde se venden comidas para llevar. Por consiguiente, tampoco en este caso ha sabido resolver de forma adecuada la traslación de este referente. En italiano un equivalente más pertinente es trattoria.

Por último, para la traslación del tradicional cap i pota amb samfaina, castellanizado por MVM en capipota con sanfaina (sic.), la traductora opta por la técnica de la adaptación. En consecuencia, reemplaza este referente por otros propios de la cultura italiana: peperonata y stufato. De todas formas, la designación propuesta no es corriente en italiano, la designación más común es stufato di vitello con peperonata.

\begin{tabular}{|r|l|}
\hline TO (p. 38) & TM (p. 39) \\
\hline $\begin{array}{r}\text { Orientó a Salvatella para que locali- } \\
\text { zara su casa de Vallvidrera. Sin ceder el }\end{array}$ & $\begin{array}{l}\text { Spiegò a Salvatella come localizzare la } \\
\text { sua casa di Vallvidrera. Senza cedere il } \\
\text { teléfono a la mujer que le urgía prisa } \\
\text { telefono alla donna che lo sollecitava } \\
\text { con tetas y ojos endurecidos por el } \\
\text { rimmel y un cruzado mágico, Carvalho } \\
\text { con le tette e con gli occhi, occhi induri- } \\
\text { tiamó a Enric Fuster, su gestor y vecino. }\end{array}$ \\
\hline
\end{tabular}

En España, el famoso modelo Cruzado Mágico de Playtex fue el protagonista del primer anuncio televisivo en el que una modelo de carne y hueso se mostraba en sujetador. Como es sabido, el primer canal de televisión nace el 28 de octubre de 1956 y el bautizo del segundo canal tiene lugar nueve años más tarde. El éxito que obtiene la pequeña pantalla es inmediato. La repercusión de todo lo que allí aparecía era inmensa ${ }^{22}$, tanto es así que la televisión asume un papel protagónico en la vida social española de aquellos años.

Esta aparente digresión surge casi espontánea al analizar esta secuen-

${ }^{21}$ Este término, rotisería, aparece ya recogido en el avance de la vigesima tercera edición del DRAE: "(Del fr. rôtisserie). 1. f. Á. R. Plata y Chile. Tienda donde se venden comidas para llevar, especialmente asados, quesos y fiambres".

${ }^{22}$ Hasta 1982, el español medio tendrá acceso a solo dos canales de televisión. En 1982 se asiste al nacimiento del tercer canal, en este caso de carácter autonómico. Para más información sobre el nacimiento y la llegada de la televisión a España, véase: < http://recursos.cnice.mec.es/media/television/bloque2/pag2.html $>$. 
cia discursiva donde la traductora, pese a la proximidad sociocultural entre España e Italia, decide eliminar este guiño del autor hacia las fórmulas de la cultura de masas. Pero, como subraya Bustos (2011: 31),

la reivindicación de la cultura popular -y de su templo: la televisión- retrata esa empatía natural hacia el pueblo llano de un autor que podía permitirse el elitismo que avalaría su vasta cultura.

Se trata además de una reducción innecesaria, pues en Italia l'incrocio magico criss cross $^{23}$ obtuvo un éxito parecido al español, aunque popularmente su denominación se abrevió en Criss Cross.

\begin{tabular}{|l|l|}
\hline TO (p. 40) & TM (p. 41) \\
\hline $\begin{array}{l}\text { Rehogó el arroz en un sofrito de } \\
\text { cebolla previamente hecho en la ca- } \\
\text { zuela. }\end{array}$ & $\begin{array}{l}\text { Affogò il riso in un soffritto di cipolla } \\
\text { preparato prima nella casseruola. }\end{array}$ \\
\hline
\end{tabular}

En esta secuencia textual la inadecuación traductiva se produce en la traslación de una comunísima técnica de cocina, rehogar. En efecto, se trata de un término que no presenta ninguna dificultad traslativa y el italiano dispone del correspondiente equivalente, rosolare. El vocablo utilizado en el TM no es adecuado, porque affogare significa literalmente en castellano ahogar.

\begin{tabular}{|c|l|}
\hline TO (p. 42) & \multicolumn{1}{|c|}{ TM (p. 43) } \\
\hline El gestor le tendió una factura por & \multicolumn{1}{|c|}{ L'amministratore gli tese una } \\
los trámites y pagos de la declaración & $\begin{array}{l}\text { ricevuta di pagamento dell'imposta } \\
\text { de renta. }\end{array}$ \\
sui redditi. \\
- ¿No te has equivocado de clien- \\
te? ¿Quieres decir que ésta no es la \\
factura de Pujol? & $\underline{\text { che questa sia la mia? }}$ \\
\hline
\end{tabular}

En cambio, en esta segmento se elide la referencia a Jordi Pujol, Presidente de la Generalidad durante 23 años (1980-2003). Es más, se efec$-$

${ }^{23}$ El modelo Cross Your Heart Bra nace en Estados Unidos en 1935. Entre las protagonistas más famosas de sus campañas figuran: Jane Russell (1971), Eve Arden (1980), Stephanie Seymour, Helena Christensen, etc. 
túa también un cambio de enfoque, pues la construcción: Sei sicuro che questa sia la mia?, literalmente en castellano equivale a: ¿Estás seguro de que esta es la mía?

Efectivamente, en la formulación original: ¿Quieres decir que ésta no es la factura de Pujol?, el apellido Pujol se erige en indicador clave que permite al lector inferir el significado que Carvalho quiere transmitir, esto es, el de una factura muy elevada. Sin embargo, con el nuevo punto de vista adoptado por la traductora, en el TM es imposible obtener la misma inferencia.

\begin{tabular}{|c|l|}
\hline TO (p. 56) & \multicolumn{1}{|c|}{ TM (p. 56-57) } \\
\hline - Entre usted en aquella cafetería & \multicolumn{1}{|c|}{-Entri in quel caffè, vedrà una ra- } \\
y verá una chica sentada [...]. & gazza seduta [...]. \\
La chica combinaba bocadito de & La ragazza alternava bocconcini di \\
porra con traguito de cortado $[\ldots]$. & brioche a piccoli sorsi di cappuccino \\
\hline
\end{tabular}

Sin duda, como señala Rabadán (1991: 164), la gastronomía es por excelencia el área de la realidad «donde se acumulan y hacen patentes las idiosincrasias culturales específicas». En esta secuencia nos hallamos, en primer lugar, frente a un vacío referencial en la LM. Se trata del término porra. En efecto, según la definición lexicográfica que nos proporciona el diccionario Clave, la porra es una: "3- Masa frita parecida al churro, pero más larga y más gruesa”. En este caso, ante el vacío léxico en la LM, la traductora opta por la técnica de la adaptación, esto es, sustituye el referente de la LO por uno análogo en la LM, aunque no cabe duda que una porra tiene muy poco que ver con una brioche, literalmente en castellano cruasán. Además, como veremos a continuación, constatamos una alternacia léxica a la hora de trasladar la palabra porra. En algunos casos se opta por el vocablo brioche, en otros se prefiere el término bignè (literalmente en castellano petisú). Algo parecido ocurre con el vocablo churro, que es trasladado indistintamente por fritella, brioche o cornetto.

Por lo que se refiere al segundo término, cortado, este no presenta ninguna dificultad traslativa y el italiano dispone de un término equivalente: caffè macchiato. En consecuencia, el uso de capuccino en el TM provoca un falso sentido innecesario. Además, este vocablo, con el sentido de "café con leche espumoso", ya aparece recogido bajo el lema 
capuchino en la vigésima primera edición del DRAE (1992).

En cuanto a la alternancia léxica, a la que nos hemos referido anteriormente a propósito de la palabra porra, presentamos a continuación varios segmentos textuales donde se evidencian una serie de alternancias para trasladar los términos churro y porra:

¿Quieres que le pregunte si los churros tienen porvenir en el Mercado Común? (TO, p. 81).

Vuoi che gli chieda se le fritelle hanno un avvenire nel Mercato Comune? (TM, p. 82).

En este ejemplo se resuelve la traslación de churro mediante una generalización, fritella, literalmente en castellano comida dulce o salada frita ${ }^{24}$. Sin embargo, más adelante cuando nos encontramos de nuevo con este referente, constatamos una incongruencia en la traslación. De hecho, la traductora opta en este caso por usar el mismo vocablo que había utilizado para trasladar el término porra:

Pero vio gente en la calle [...] y creyó oler perfume de churro, oír el claqué de las tazas de café sobre los platillos. Se duchó (TO, p. 117).

Ma vide gente per la strada [...] e credette di sentire un profumo di brioches, di udire il tintinnio delle tazze di caffè sui piattini. Si fecce una doccia (TM, p. 116).

Por último, encontramos una secuencia textual donde aparecen los dos referentes gastronómicos: porra y churro. Aquí la traductora ofrece una nueva solución para trasladar estos elementos, esto es, petisú y crusán:

Se levantó, se vistió, recorrió un aburrido horizonte de porras, churros y cortados sobre los mostradores de las madrugadoras cafeterías del barrio [...] (TO, p. 227).

Si alzò, si vestì; passò in rassegna un noioso orizzonte di bignè, cornetti e cappuccini sui banconi dei mattinieri caffè del quartiere [...] (TM, p. 222).

En realidad, por lo que se refiere al término churro, consideramos que

${ }^{24}$ En el Diccionario bilingue Zanichelli se recoge como equivalente italiano de churrro el vocablo fritella, pero también se nos da como equivalente del término castellano buñuelo el vocablo fritella. En realidad, la fritella, como se apunta en el Treccani es: "s. f. [der. di fritto]. - 1. (gastron.) [vivanda dolce o salata che si prepara facendo friggere in padella una piccola porzione di pasta semiliquida, spesso unita ad altri ingredienti]”. 
se trata de una palabra que, al igual que paella, sangría, chorizo, etc., se ha impuesto ya internacionalmente en la forma correspondiente a la LO. Por tanto, creemos que es más acertado mantener el préstamo puro en el LM.

En líneas generales, por lo que se refiere a la traslación de los realia gastronómicos, nos parece una solución sensata recurrir al préstamo. Porque el préstamo, como observa Rabadán (1991: 167), permite conservar la asociación signo-referente sin tener que recurrir a la técnica de la amplificación mediante paráfrasis explicativas, notas del traductor, etc. ${ }^{25}$

\begin{tabular}{|l|l|}
\hline TO (p. 65) & \multicolumn{1}{|c|}{ TM (p. ) } \\
\hline - ¿Y ése quién es? [...]. & - E quello chi è? [...]. \\
- Romanones. & - $\underline{\text { Garibaldi. }}$ \\
\hline
\end{tabular}

En esta secuencia discursiva asistimos a la sustitución de un personaje histórico, el conde de Romanones (1863-1950), por otro de la cultura receptora, Giuseppe Garibaldi (1807-1882). Resulta un tanto arbitrario este intercambio, porque aparte de la dedicación a la política, poco tienen que ver ambos personajes.

\begin{tabular}{|c|c|}
\hline TO (p. 123) & TM (p. 122) \\
\hline $\begin{array}{l}\text { Cogió un taxi y le pidió que le } \\
\text { llevara a la calle Profesor Waksman. } \\
\text { - A lo mejor llegamos antes del fi- } \\
\text { nal de la Liga, porque hay un parón de } \\
\text { no te menees, es que no pue'ser, no } \\
\text { pue'ser. }\end{array}$ & $\begin{array}{l}\text { Prese un taxi e chiese d'esser portato } \\
\text { in via Profesor Waksman. } \\
\text { - Può darsi che arriviamo prima che } \\
\text { finisce questo raduno, perché c'è un } \\
\text { ingorgo che non vi dico, non se ne } \\
\text { può più, non si può proprio andare } \\
\text { avanti così. }\end{array}$ \\
\hline
\end{tabular}

Aquí, la traductora no sabe resolver adecuadamente la traslación del término liga ${ }^{26}$,competición de sobra conocida en ambas culturas y que encuentra su equivalente en la denominación italiana campionato. Sin

$-$

${ }^{25}$ Para Rabadán (1991: 167) se trata de la opción "menos mala", pues como subraya la autora, no obliga a la explicitación de todos y cada uno de los rasgos léxicos que componen la unidad en el TO.

${ }^{26}$ La definición que proporciona el DRAE es: "competición deportiva en que cada uno de los equipos admitidos ha de jugar con todos los de su categoría”. 
embargo, en la LM selecciona el vocablo raduno (literalmente en castellano reunión). Por consiguiente, la técnica de la creación discursiva o equivalencia efímera, en este caso concreto, no es acertada. Nótese también como para caracterizar diastráticamente al taxista MVM evidencia la pérdida de la $d$ intervocálica, pérdida típica de hablas populares o regionales, especialmente meridionales.

\begin{tabular}{|c|c|}
\hline TO (p. 128) & TM (p. 127-128) \\
\hline $\begin{array}{l}\text { - En la Gran Tasca ponen cocido } \\
\text { hoy. Gracias a ti me estoy enterando } \\
\text { de cada cosa. }\end{array}$ & $\begin{array}{l}-\mathrm{Al} \text { «Tavernone» fanno il lesso, oggi. } \\
\text { Grazie a te mi sto informando di tutto. }\end{array}$ \\
\hline
\end{tabular}

Al margen de la libertad del traductor, creemos que hay ocasiones en que lo que prima es el término original, sobre todo cuando se trata del nombre de restaurantes, calles, etc. Porque, ciertamente será harto difícil que un italiano que vaya a Madrid logre localizar el Tavernone, tarea más fácil será si pregunta por la Gran Tasca ${ }^{27}$.

Una consideración muy parecida nos merece la traducción del término cocido. Según la lexicografía italiana, el lesso es un plato de carne hervida ${ }^{28}$. En cambio, ya sólo la definición lexicográfica que nos proporciona el diccionario Clave de cocido: "Guiso preparado con garbanzos, carne, tocino y hortalizas", presenta diferencias abismales con respecto a la de lesso.

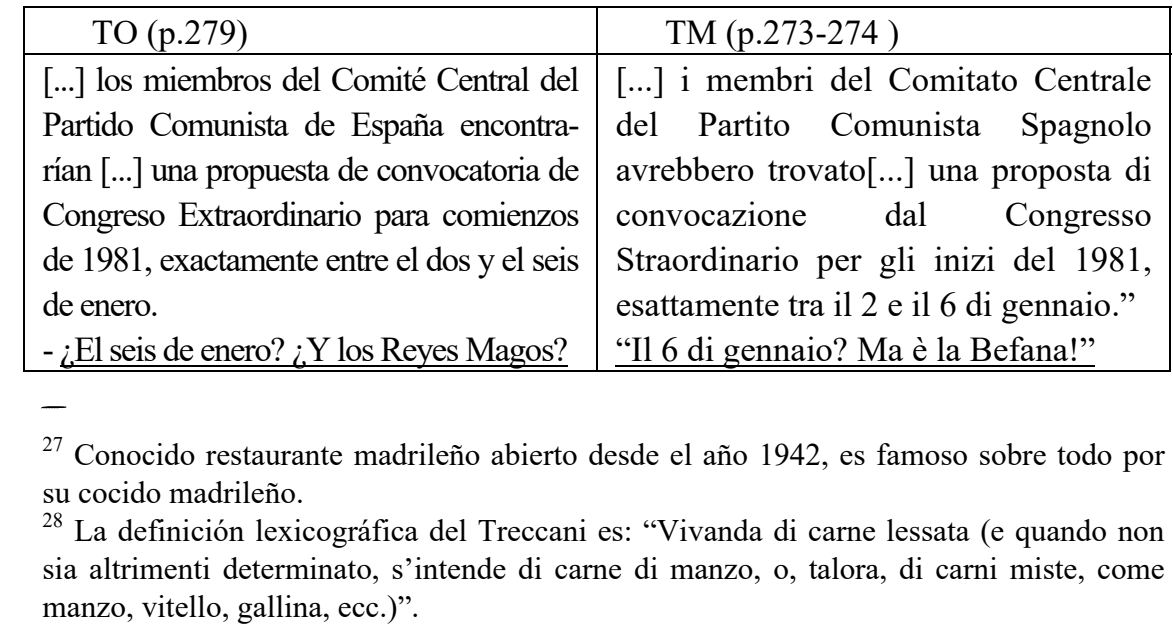


En Italia, en vísperas de la Epifanía es la Befana, "vecchia, bruttissima ma benefica, che di notte, scendendo per la cappa del camino, lascia nelle scarpe, o più spesso nelle calze, dei bambini buoni, doni e dolciumi (ai cattivi, pezzi di carbone)" (Treccani) quien ejerce como nuestros Reyes Magos o como Santa Claus es los países nórdicos de generosa portadora de regalos a los niños. Grosso modo, ${ }^{29}$ nos hallamos ante una misma traducción pero con protagonistas diferentes. La traductora, frente al escollo que representa este elemento cultural, opta por la técnica de la adaptación sustituyendo los Reyes Magos por la Befana. De hecho, en el siguiente segmento mantiene también el mismo criterio.

\begin{tabular}{|l|l|}
\hline \multicolumn{1}{|c|}{ TO (p.279) } & \multicolumn{1}{|c|}{ TM (p. 274) } \\
\hline Leveder pedía explicaciones a todos & Leveder chiedeva spiegazioni a tutti i \\
los miembros del Comité Ejecutivo & membri del Comitato Esecutivo che \\
que encontraba entre los grupos. & incontrava tra i vari gruppi. \\
- ¿Cómo vamos a normalizar nuestra & - Come normalizzeremo il nostro \\
relación con la sociedad si no podemos & rapporto con la società se non \\
compartir con nuestros hijos la alegría & possiamo dividere con i nostri figli la \\
de recibir los juguetes de manos de Sus & gioia di ricevere i giocattoli nel giorno \\
\hline$\underline{\text { Majestades los Reyes Magos? }}$ & $\underline{\text { della Befana? }}$ \\
- Anda ya. & - Smettila. \\
- Pues a más de uno le va a zurrar la & - Più di uno se la dovrà vedere con \\
badana la parienta, porque ya es el & sua moglie, perché è il colmo che \\
colmo que hasta el día de Reyes se & anche il giorno della Befana si debba \\
tenga que hacer política. & fare politica. \\
- Mi mujer me pregunta si estoy casa- & - Mia moglie mi chiede se sono \\
do con ella o con el partido. & sposato con lei o con il partito. \\
\hline
\end{tabular}

Sin embargo, en el segmento que presentamos a continuación, la segunda vez que aparece el referente Reyes, la traductora recurre a un doblete de técnicas: traducción literal y amplificación. Desde nuestro punto de vista, esta elección no puede sino confundir al lector meta, porque hasta este momento se ha hecho referencia sólo a la Befana.

\footnotetext{
${ }^{29}$ En Italia, dependiendo de las zonas, ejercen de portadores de regalos: Santa Lucia (Bergamo, Verona), Babbo Natale y La Befana.
} 


\begin{tabular}{|l|l|}
\hline \multicolumn{1}{|c|}{ TO (p.279) } & \multicolumn{1}{|c|}{ TM (p. 274) } \\
\hline Leveder iba provocando pequeñas & Leveder andava provocando piccole \\
tempestades dialécticas. & tempeste dialettiche. \\
- Mir. Tengo una idea para resolver el & - Mir. Ho un'idea per risolvere il \\
problema del día de Reyes. & problema del giorno della Befana. \\
- Para mí no es ningún problema. & - Per me non c'è nessun problema. \\
- ¿Y los niños? Esperan ilusionados el & - Ed i bambini? Aspettano ansiosi i \\
regalo de Reyes. & doni dei Re Magi. \\
- Tengo a los hijos crecidos. Y ade- \\
$\begin{array}{l}\text { más son republicanos desde que na- } \\
\text { cieron. }\end{array}$ & repubblicani dalla nascita. E poi sono \\
\hline
\end{tabular}

Aquí también se repite el mismo dualismo traslativo que hemos verificado en el texto precedente. Consideramos que se hubiera podido evitar la ambigüedad que esta opción provoca en el TM seleccionando una de las dos soluciones y manteniéndola coherentemente a lo largo de todo el texto. De hecho, la introducción del término Re Magi en el TM parece casi un tentativo in extremis para preservar el juego de palabras y la ironía que se crea entre ir vestido de negro, es decir, disfrazado de Rey Baltasar, y la inferencia trabajar como un negro.

\begin{tabular}{|c|c|}
\hline TO (p.280) & TM (p. 274-275) \\
\hline $\begin{array}{l}\text { Contestó Mir a Sepúlveda Civit. En } \\
\text { un corro reían estruendosamente al- } \\
\text { gún comentario de Leveder. } \\
\text { - Mir. Acércate, se habla de ti. } \\
\text { - ¿Qué ha dicho de mí este euroanar- } \\
\text { co? } \\
\text { - Propone que el día de Reyes vengan } \\
\text { nuestros hijos a la sede del Congreso } \\
\text { y tú les des los juguetes vestido de } \\
\text { Rey Mago. } \\
\text { - Buena idea. De negro. Eso es lo que } \\
\text { he hecho toda mi vida. De negro. Lo } \\
\text { propondremos al final. }\end{array}$ & $\begin{array}{l}\text { Mir rispose a Sepúlveda Civit. In un } \\
\text { grupetto si rideva fragorosamente di } \\
\text { un certo commento di Leveder. } \\
\text { - Che dice di me quell'euroanarchico? } \\
\text { - Sta proponendo che il giorno della } \\
\text { Befana portiamo alla sede del } \\
\text { Congreso i figli e tu gli consegni i } \\
\text { giocattoli vestito da uno dei Magi. } \\
\text { - Buona idea. Il negro. Ho fatto questo } \\
\text { per tutta la vita, il negro. Lo propo- } \\
\text { rremo alla fine. }\end{array}$ \\
\hline
\end{tabular}


En este último segmento, nos hallamos de nuevo con referentes culinarios, ámbito extremadamente prolífico y recurrente en la producción montalbaniana.

\begin{tabular}{|c|c|}
\hline & \\
\hline $\begin{array}{l}\text { (...) Carvalho propuso tomar algo, } \\
\text { una cerveza por ejemplo, le propuso a } \\
\text { la antialcohólica Carmela. Águila } \\
\text { siempre fresquita con su sabor tan } \\
\text { natural, canturreó ella. } \\
\text { - ¡Marchando dos cañas! ¡Y una } \\
\text { empanada de lomo! } \\
\text { - ¿Será buena esa empanada? } \\
\text { - Es simbólica. Es un monumento } \\
\text { al lomo desconocido. }\end{array}$ & $\begin{array}{l}\text { (...) Carvalho propose di bere } \\
\text { qualcosa, una birra per esempio, alla } \\
\text { antialcolica Carmela. Birra sempre } \\
\text { fresca nel suo gusto naturale, } \\
\text { canticchiò lei. } \\
\text { - Subito due birre alla spina! E una } \\
\text { braciola di maiale! } \\
\text { - Sarà buona la braciola? } \\
\text { - È simbolica. È un monumento al } \\
\text { maiale sconosciuto. }\end{array}$ \\
\hline
\end{tabular}

En primer lugar, para trasladar el nombre comercial de una conocida marca de cerveza, Águila, la traductora opta por el hiperónimo birra, literalmente en castellano cerveza, difuminando por tanto el referente.

En segundo lugar, se produce una inadecuación de traducción al trasladar empanada de lomo por braciola di maiale, literalmente en castellano chuleta cerdo.

Tampoco es adecuada la solución propuesta en el TM para lomo, pues maiale, en castellano es literalmente cerdo, el término equivalente en italiano de lomo es lonza.

\section{A modo de conclusión}

Los segmentos textuales que acabamos de analizar evidencian de forma significativa la actitud tomada por la traductora con respecto a los elementos culturalmente marcados del ámbito del patrimonio cultural. En efecto, se nota ya desde las primeras páginas la aclimatación del TO a la cultura meta. Ante este manifiesto etnocentrismo respecto a los elementos culturales foráneos, cabe preguntarse si realmente para los lectores italianos los referentes que acabamos de reseñar resultan verdaderamente tan ajenos, tan opacos como para que sea necesario recurrir casi sistemáticamente en el TM a términos más generales o incluso a la naturalización completa de estos a la cultura receptora. 
En líneas generales, consideramos un buen criterio mantener los términos originales cuando se trata de platos típicos, monumentos emblemáticos, personajes históricos, etc. Además, como apuntan los estudios más recientes en traductología, el acto traslativo se fundamenta no sólo en el buen dominio por parte del traductor de su lengua materna (LM) y del idioma de trabajo (LO), sino también en un buen conocimiento del asunto o tema del texto. Sin duda, esta última competencia deviene imprescindible sobre todo cuando nos hallamos ante obras tan ricas culturalmente como es la serie carvalhiana. ${ }^{30}$

A nuestro modo de ver, y de acuerdo con Esther Benítez (1995: 116), en la traducción literaria es muy importante "que el lector, cuando entra en la historia, sepa perfectamente que se desarrolla en otro país, con otra cultura y otros usos y costumbres" ${ }^{\prime 31}$.

Efectivamente, consideramos que en la traslación de un texto literario es indispensable que el traductor comprenda y reproduzca toda la información contenida en el TO. Por consiguiente, para poder llevar a cabo el trasvase lingüístico, cultural y artístico que conlleva el TO es imprescindible que el traductor conozca en profundidad las dos lenguas y las dos culturas. Solo así podrá evitar daños esenciales en la trasmisión de la obra literaria, porque, en buena medida, solo de él dependerá la imagen que se proyecte en la lengua-cultura meta del texto y del autor que está traduciendo.

\section{Bibliografía}

Arqués Corominas, R. y Padoan, A. Il Grande dizionario di spagnolo. Dizionario spagnolo-italiano, italiano-spagnolo. Milano: Zanichelli, 2012.

Arroyo, F. "Carvalho es una solución técnica" en El País. Madrid: 07 de

\section{$-$}

${ }^{30}$ De hecho, el propio MVM reconoce en la larga conversación que mantiene con Tyras (2003: 122) que su obra está llena de juegos, guiños y tics, "que sólo entiende una minoría de lectores. Pero es normal porque la novela se escribe para muchos sedimentos y cada uno de ellos te lee a un nivel y percibe una (sic.) cosas que otro sedimento no recoge". Esta peculiaridad obliga a una atenta traslación a fin de que el TM cumpla también el mismo cometido en la lengua-cultura de llegada.

${ }^{31}$ Como subraya Colmeiro (1994: 171), MVM "para hacer su crónica de la vida colectiva española durante y después de la transición democrática" recurre a unos personajes tipo, a la gastronomía, a las canciones populares, y especialmente a los diversos lenguajes de los distintos grupos sociales dentro de sus respectivas comunidades. 
abril de 1983.

Benítez, E. "Pentimento: Un relato de Alberto Moravia 20 años después» en Marco Borillo, J (ed.). La traducció literaria, Castello: Universidad Jaume I, Servicio de Comunicación y Publicaciones, 1995, 107116.

Bensoussan A. , "El traductor en la noche oscura del sentido" en Donaire Fernández, M. L. y F. Lafarga (eds.). Traducción y adaptación cultural: España-Francia. Universidad de Oviedo: Servicio de Publicaciones, 1991, 15-20.

Bustos, J. "El periodismo epocal de Vázquez Montalbán" en Revista de libros. Madrid: $\mathrm{n}^{\circ}$ 170, febrero de 2011: 31 .

Canals, E. "Vázquez Montalbán: 'La novela es una crítica al sentido religioso de la militancia"” en El País. Barcelona: 07 de abril de 1981.

CLAVE. Diccionario de uso del español actual. Madrid: SM, 2006.

Colmeiro, José F., El ruido y la furia. Conversaciones con Manuel Vázquez Montalbán, desde el planeta de los simios, Madrid: Iberoamericana/Vervuert, 2013.

. La Novela Policíaca Española. Teoría e historia crítica, Barcelona: Anthropos, 1994.

DEA. Diccionario del español actual. Madrid: Aguilar, 1999.

García Montero, L. "La suciedad y la compasión". En Vázquez Montalbán, M. Puente aéreo, Barcelona: Planeta, 2012. 7-14.

García Yebra, V. Traducción: historia y teoría. Madrid: Gredos, 1994.

Genette, G. Umbrales. México: Siglo XXI, 2001.

Grupo de Investigación HUM-807, "La angustia y el gozo de traducir. Entrevista a Miguel Martínez-Lage" en Odisea, n. 8, 2007. 215-221.

Guerriero, L. (2013), "El alma de los libros" en El País Babelia. Madrid: 29 de junio de 2013. 5-6.

Hurtado Albir, A. Traducción y traductología. Madrid: Cátedra, 2004 [2001].

Molina Martínez, L. El otoño del pingüino. Castelló de la Plana: Universitat Jaume I, 2006.

Montalto Cessi, D. "Uno specchio per i testi: l'epigrafe letteraria nel romanticismo spagnolo" en Culture. Milano, 1989.

. Uno specchio per i testi: l'epigrafe letteraria in Pío Baroja. Milano: Marcos y Marcos, 1990. 
Newmark, P. Manual de traducción. Madrid: Cátedra, 2004 [1992].

Obiols, I. y P. Ferrer, El mercado de la Boquería. Barcelona: Península, 2004.

Pradera, J. "Pepe Carvalho cabalga de nuevo" en El País. Madrid: 26 de abril de 1981.

Rabadán, R. Equivalencia y traducción (Problemática de la equivalencia translémica inglés-español). León: Universidad de León, 1991.

RAE. Diccionario de la Lengua Española. En http://lema.rae.es/drae/.

Rodríguez Abella R. M. "El hombre de mi vida: análisis de la traducción de los culturemas del ámbito gastronómico" en La comunicación especializada. Ed. Navarro C., R. M. Rodríguez Abella, F. Dalle Pezze y R. Miotti. Berna: Peter Lang, 2008. 319-355.

Rodríguez Abella R. M. "Oralidad y traducción. (Reflexiones en torno a la traducción de los diálogos en una novela de la serie Carvalho)" en Vivir es volver. Studi in onore di Gabriele Morelli. Ed. Bernad M., I. Rota y M. Bianchi. Bergamo: Sestante edizioni, 2009. 427-436. . "Viaje al universo Carvalho (Los pájaros de Bangkok: referencias culturales y traducción)" en Viaggiare con la parola. Ed. Canals J. y E. Liverani. Milano: FrancoAngeli, 2010. 165-182. . "Un delitto per Pepe Carvalho: referencias culturales y traducción" en MVM: Cuadernos de Estudios Manuel Vázquez Montalbán, vol. 1, 2013. 63-86.

Sabia, S. "Paratexto. Títulos, dedicatorias y epígrafes en algunas novelas mexicanas" en Espéculo. Revista de estudios literarios. Madrid: Universidad Complutense de Madrid, $2005 . \quad$ URL: http://www.ucm.es/info/especulo/numero31/paratext.html. (07/01/2014).

Saval, José V., Manuel Vázquez Montalbán. El triunfo de un luchador incansable, Madrid: Editorial Síntesis, 2004.

Tyras, Georges, Geometrías de la memoria. Conversaciones con Manuel Vázquez Montalbán, Granada: Zoela ediciones, 2003.

Vázquez Montalbán, M. Asesinato en el Comité Central, Barcelona: Planeta, 1997 [1981].

- Assassinio al Comitato Centrale, Palermo: Sellerio editore (Traducción de Lucrecia Panunzio Cipriani), 1984.

. Las recetas de Carvalho, Barcelona: Planeta, 2004 [1989].

Vocabolario Treccani. En http://www.treccani.it/vocabolario [17.09.2013] 